\title{
Downregulation of ubiquitin-specific peptidase 39 suppresses the proliferation and induces the apoptosis of human colorectal cancer cells
}

\author{
ZHIYUAN XING ${ }^{1}$, FENGBO SUN ${ }^{2}$, WANG HE$^{3}$, ZHIWEI WANG $^{1}$, XIUQI SONG $^{1}$ and FENGJUAN ZHANG ${ }^{4}$ \\ ${ }^{1}$ Department of General Surgery, The Second Affiliated Hospital of Qingdao University Medical College; \\ ${ }^{2}$ Department of General Surgery, Qingdao Haici Medical Group; ${ }^{3}$ Department of Hepatopathy, \\ Qingdao Sixth People's Hospital; ${ }^{4}$ Department of Infection, The Second Affiliated Hospital of \\ Qingdao University Medical College, Qingdao, Shandong 266000, P.R. China
}

Received October 13, 2016; Accepted October 20, 2017

DOI: $10.3892 / \mathrm{ol} .2018 .8061$

\begin{abstract}
Ubiquitin-specific peptidase 39 (USP39) has been reported to participate in the mitotic spindle checkpoint and the process of cytokinesis. and has been identified as a therapeutic target for various types of cancer. However, the effect of USP39 in colorectal cancer (CRC) has not been investigated. To explore the functional role of USP39 in CRC cell growth, lentivirus-mediated RNA interference was applied to inhibit USP39 expression in SW1116 and HCT116 cells. The relative USP39 mRNA and protein expression levels were significantly reduced in the USP39 knockdown cells, as verified by reverse transcription-quantitative polymerase chain reaction and western blot analysis. USP39 knockdown significantly reduced the proliferation and colony formation abilities of CRC cells, and induced apoptosis and cell cycle arrest in the $\mathrm{G}_{2} / \mathrm{M}$ phases, as determined by an MTT assay, a colony formation assay and flow cytometry analysis. Furthermore, western blot analysis demonstrated that USP39 knockdown may have induced apoptosis through the upregulation of p53, p-p53, PARP and caspase-3 expression in SW1116 cells. In conclusion, USP39 may be a novel biological marker for targeted therapy against CRC, and requires further investigation.
\end{abstract}

\section{Introduction}

Colorectal cancer (CRC) is one of the most fatal types of cancer worldwide (1). A range of chemotherapeutic drugs

Correspondence to: Dr Fengjuan Zhang, Department of Infection, The Second Affiliated Hospital of Qingdao University Medical College, 1677 Mount Wutai Road, Qingdao, Shandong 266000, P.R. China

E-mail: 18661802286@163.com

Key words: colorectal cancer, ubiquitin-specific peptidase 39, small hairpin RNA, cell apoptosis, targeted therapy and other methods are available for the treatment of CRC, but the associated side effects hamper their clinical application and efficacy (2). With the development of molecular-targeted therapies, progress has been made in cancer therapy. In recent years, a number of molecules have been identified as targets for molecular-targeted anticancer drugs, including growth factors, factors regulating cell survival and molecules associated with the cell cycle (3). There is a critical need to identify potential molecular targets and improve the therapy of CRC.

Ubiquitin-specific peptidase 39 (USP39) is a deubiquitinating enzyme containing a central zinc finger and two ubiquitin C-terminal hydrolase domains (4). USP39 is critical in the process of pre-mRNA splicing, but does not exhibit ubiquitin-specific peptidase activity (5). As a $65 \mathrm{kDa}$ SR protein of the U4/U6.U5 tri-small nuclear ribonucleoprotein complex, USP39 also functions in the assembly of the mature spliceosome complex and is indispensable in maintaining the integrity of the mitotic spindle checkpoint $(6,7)$. A previous study indicated that USP39 silencing induced defective chromosome segregation and cytokinesis in U2OS cells, indicating USP39 is critical in the regulation of mitosis (7). Furthermore, the pro-growth effect of USP39 in cancer cells has been widely investigated. Wen et al (8) demonstrated that the overexpression of USP39 promoted the proliferation of prostate cancer cells, which is in accord with a study by Wang et al (9) in breast cancer cells. Furthermore, USP39 knockdown has been demonstrated to affect cell cycle distribution by inducing arrest in the $\mathrm{G}_{2} / \mathrm{M}$ phase, and to promote apoptosis, in human hepatocellular carcinoma (10) and medullary thyroid carcinoma (11) cells, indicating that USP39 may be a feasible target for the molecular therapy of various types of cancer. However, limited data regarding the biological function of USP39 in CRC cells is available.

Therefore, the effect of USP39 in CRC cells was assessed in the present study. Lentivirus-delivered short hairpin RNA (shRNA) was used to infect CRC cells to facilitate loss-of-function analysis. The function of USP39 knockdown on the proliferation, colony formation ability, cell cycle progression and apoptosis of CRC cells was 
investigated. To the best of our knowledge, this is the first study to demonstrate that USP39 is associated with CRC cell proliferation.

\section{Materials and methods}

Cell lines and cell culture. SW1116 and HCT116 human CRC cells, and 293T cells were purchased from the Cell Bank of Chinese Academy of Sciences (Shanghai, China). SW1116 and 293T cells were cultured in Dulbecco's modified Eagle's medium (DMEM; Gibco; Thermo Fisher Scientific, Inc., Waltham, MA, USA) with $10 \%$ fetal bovine serum (FBS; Gibco; Thermo Fisher Scientific, Inc.). HCT116 cells were cultured in RPMI-1640 (Hyclone; GE Healthcare Life Sciences, Logan, UT, USA) supplemented with 10\% FBS. All cells were maintained at $37^{\circ} \mathrm{C}$ in a humidified incubator with $5 \% \mathrm{CO}_{2}$.

Construction of lentiviral particles and USP39 knockdown. To silence the expression of USP39, two candidate shRNAs for human USP39, based on the Gen Bank sequence NM_001256725.1, were designed (KD-1 and -2). The shRNA sequences targeting USP39 were as follows: KD-1, 5'-GAT TTGGAAGAGGCGAGATAACTCGAGTTATCTCGCCTC TTCCAAATC-3'; KD-2, 5'-CCTTCCAGACAACTATGAGA TCTCGAGATCTCATAGTTGTCTGGAAGG-3'. A non-silencing shRNA with the sequence, 5'-TTCTCCGAACGTGTC ACGTCTCGAGACGTGACACGTTCGGAGAA-3', was used as a negative control (shCon). The oligos were annealed and inserted into pFH-L plasmids (Shanghai Holly Lab, Shanghai, China), which included a green fluorescent protein (GFP) tag. The lentiviral particles were constructed in 293T cells as described in a previous study (12). SW1116 and HCT116 cells were incubated in 6-well plates and infected with KD or shCon for $96 \mathrm{~h}$ with a multiplicity of infection of 30 . Successful infection was confirmed by the observation of GFP expression with fluorescence microscopy (DMI4000B; Leica Microsystems $\mathrm{GmbH}$, Wetzlar, Germany). The efficiency of knockdown was determined by reverse transcription-quantitative polymerase chain reaction (RT-qPCR) and western blot analyses. All experiments were repeated in triplicate.

RT-qPCR analysis. Cells were harvested at 5 days after lentiviral infection. TRIzol reagent (Thermo Fisher Scientific, Inc.) was used to extract total RNA, according to the manufacturer's instructions. Single-stranded cDNA was synthesized using Superscript II Reverse Transcriptase (Invitrogen; Thermo Fisher Scientific, Inc.). The primers were as follows: USP39 forward, 5'-GCCAGCAGAAGAAAAAGAGC-3' and reverse, 5'-GCCATTGAACTTAGCCAGGA-3'; $\beta$-actin (endogenous control) forward, 5'-GTGGACATCCGCAAAGAC-3' and reverse, 5'-AAAGGGTGTAACGCAACTA-3'. The mRNA levels of USP39 were determined using SYBR Green on the Bio-Rad Connect Real-Time PCR system (Bio-Rad Laboratories, Inc., Hercules, CA, USA). The total PCR reaction volume was $20 \mu 1$, including $10 \mu \mathrm{l} 2 \mathrm{X}$ SYBR Premix Ex Taq (Takara Bio, Inc., Otsu, Japan), $0.5 \mu \mathrm{l}$ primers $(2.5 \mu \mathrm{M}), 5 \mu \mathrm{l}$ cDNA and $4.5 \mu \mathrm{ldd} \mathrm{H}_{2} \mathrm{O}$. The PCR thermocycling procedure was as follows: Initial denaturation at $95^{\circ} \mathrm{C}$ for $1 \mathrm{~min}$, followed by 40 cycles of denaturation at $95^{\circ} \mathrm{C}$ for $5 \mathrm{sec}$ and extension at $60^{\circ} \mathrm{C}$ for $20 \mathrm{sec}$. The relative gene expression levels were calculated and compared using the $2^{-\Delta \Delta \mathrm{Cq}}$ method (13).

Western blot analysis. Cells were harvested and lysed in $2 \mathrm{X}$ protein lysis buffer [10 mM EDTA, $100 \mathrm{mM}$ Tris- $\mathrm{HCl}$ (pH 6.8), 4\% SDS and $10 \%$ glycine] 5 days after lentiviral infection. Protein lysates were collected by centrifugation at $12,000 \mathrm{x} g$ for $15 \mathrm{~min}$ at $4^{\circ} \mathrm{C}$. Equal amount of protein samples $(30 \mu \mathrm{g})$ were separated on $10 \%$ SDS-PAGE and transferred to a PVDF membrane at $300 \mathrm{~mA}$ for $1.5 \mathrm{~h}$. Then the membrane was blocked with Tris-buffered saline with $0.1 \%$ Tween-20 containing 5\% non-fat dried milk for $1 \mathrm{~h}$ at room temperature, and probed with the corresponding primary antibodies. Primary antibodies included mouse anti-p53 (dilution 1:1,000; sc-126; Santa Cruz Biotechnology, Inc., Dallas, TX, USA), rabbit anti-p-p53 (dilution 1:500; cat. no. 2528), rabbit anti-caspase-3 (dilution 1:500; cat. no. 9661), rabbit anti-poly(ADP-ribose) polymerase (PARP; dilution 1:1,000; cat. no. 9542; all from Cell Signaling Technology, Inc., Danvers, MA, USA) and rabbit anti-GAPDH (dilution 1:100,000; cat. no. 10494-1-AP; Protein Tech Group, Inc., Chicago, IL, USA). The membranes were immunoblotted with the appropriate primary antibodies at $4^{\circ} \mathrm{C}$ overnight, and then incubated with a HRP-conjugated goat anti-rabbit (dilution 1:5,000; cat. no. SC-2054) or goat anti-mouse antibody (dilution 1:5,000; cat. no. SC-2005; both from Santa Cruz Biotechnology, Inc.) as appropriate for $2 \mathrm{~h}$ at room temperature. Signals were detected using Super ECL Detection Reagent (Applygen Technologies, Inc., Beijing, China).

MTT assay for cell viability. SW1116 and HCT116 cells were seeded at 2,000 cells/well in 96-well plates at 4 days after infection. MTT solution $(20 \mu \mathrm{l})$ was added into each well, and incubated for a further $4 \mathrm{~h}$ at $37^{\circ} \mathrm{C}$. The supernatant was removed and $100 \mu \mathrm{l}$ acidic isopropanol (10\% SDS, 5\% isopropanol and $0.01 \mathrm{~mol} / \mathrm{l} \mathrm{HCl}$ ) was added to each well. The absorbance values at $595 \mathrm{~nm}$ were determined using a microplate reader.

Colony formation assay. Stably transfected SW1116 (500 cells/well) and HCT116 (400 cells/well) cells were seeded into 6-well plates. Following incubation for 8 days (SW1116) or 7 days (HCT116), cells were washed in ice-cold PBS and fixed with methanol at the temperature of $37^{\circ} \mathrm{C}$ for $15 \mathrm{~min}$. Then crystal violet (Beyotime Institute of Biotechnology, Haimen, China) staining was performed, according to the manufacturer's instructions. The total number of colonies, defined as groups of $>50$ cells, was counted under light microscopy. The colony images were analyzed using Metamorph software version 7.5 (Molecular Devices LLC, Sunnyvale, CA, USA).

Cell cycle analysis. To investigate the mechanisms underlying the effects of USP39 on cell growth, cell cycle distribution was examined by flow cytometry and propidium iodide (PI) staining. SW1116 (7x10 ${ }^{4}$ cells/well) and HCT116 ( $2 \times 10^{5}$ cells/well) cells were harvested at 7 days and 5 days after infection, respectively. Cells were fixed in $70 \%$ ice-cold ethanol for $4 \mathrm{~h}$ and washed twice with cold PBS. The fixed cells were stained with PI and detected by a FACSCalibur flow cytometer (BD Biosciences, San Jose, CA, USA) according to 
SW1116

Con

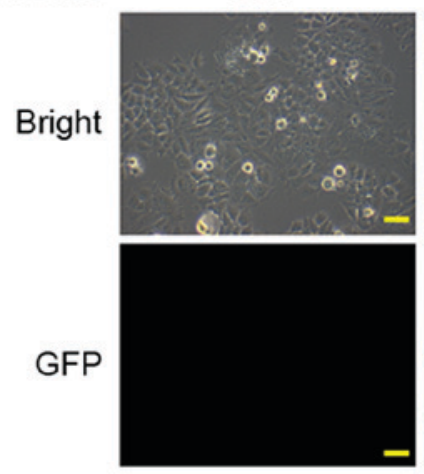

HCT116
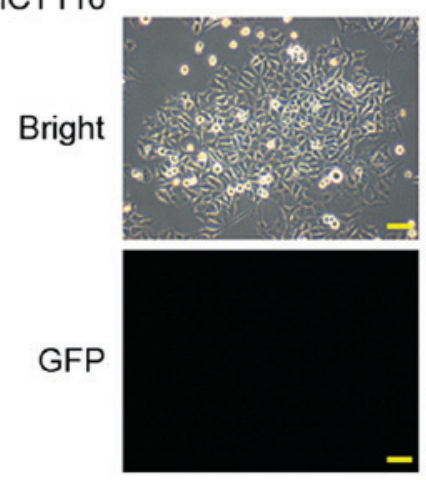

shCon
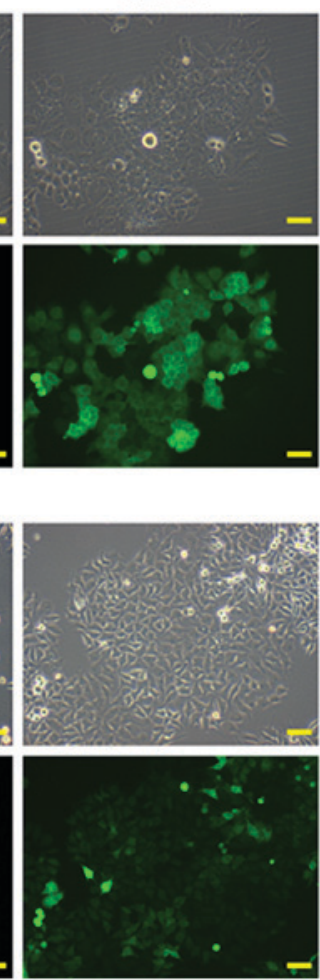

KD-1
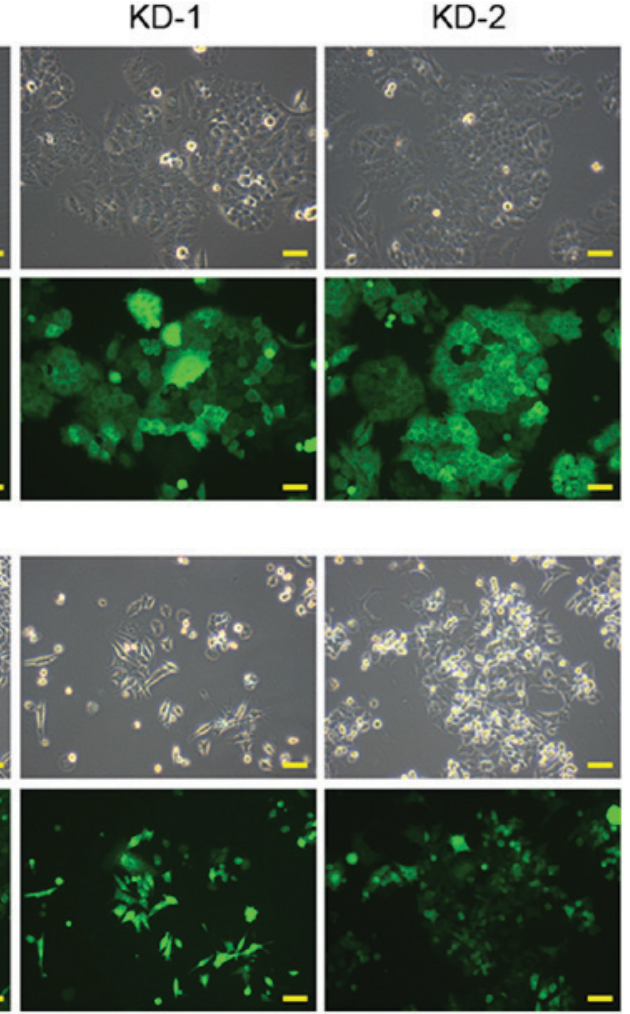

Figure 1. Representative images of lentivirus infection efficiency in SW1116 and HCT116 cells as determined by GFP expression. GFP, green fluorescent protein; Con, untransfected control; shCon, control small hairpin RNA; KD-1, first small hairpin RNA against ubiquitin-specific peptidase 39; KD-2, second small hairpin RNA against ubiquitin-specific peptidase 39. Scale bar, $100 \mu \mathrm{m}$.

the manufacturer's protocol. The results were analyzed using FlowJo software version 7.6.2 (FlowJo LLC, Ashland, OR, USA).

Apoptosis analysis. To further confirm whether the effect of USP39-knockdown on cell proliferation was associated with apoptosis, flow cytometry with Annexin V-APC/7-AAD double staining was performed on SW1116 cells. Briefly, at 7 days after infection, SW1116 cells $\left(8 \times 10^{4}\right.$ cells/dish) were seeded in $6-\mathrm{cm}$ dishes and cultured for a further $48 \mathrm{~h}$. Then the cells were collected, double stained using Annexin V-APC and 7-AAD according to the manufacturer's protocol (Nanjing Key Gen Biotech Co., Ltd., Nanjing, China), and detected by a FACSCalibur flow cytometer.

Statistical analysis. All experiments were repeated in triplicate and the results were presented as the mean \pm standard deviation of three independent experiments using GraphPad Prism 5 (GraphPad Software, Inc., La Jolla, CA, USA). Analysis was performed with a one-way analysis of variance followed by Dunnett's multiple comparisons test. $\mathrm{P}<0.05$ was considered to indicate a statistically significant difference.

\section{Results}

Lentivirus-mediated RNAi suppresses the expression of USP39. To investigate the effects of USP39 in CRC, the expression of USP39 was knocked down in SW1116 and HCT116 cells using lentivirus-mediated transfection. As shown in Fig. 1,
$>80 \%$ of the cells expressed GFP following infection with shRNA targeting USP39 (KD-1 and -2) or control shRNA (shCon), suggesting that the recombinant lentivirus generated high infection efficiency in CRC cells. RT-qPCR analysis indicated that the USP39 mRNA levels were significantly downregulated in the KD-1 and 2 groups compared with the shCon and Con groups of SW1116 and HCT116 cells (Fig. 2A; $\mathrm{P}<0.0001)$. Consistent with this, USP39 protein levels were also downregulated in the KD-1 and -2 groups of SW1116 and HCT116 cells (Fig. 2B). Therefore, it was demonstrated that both shRNAs against USP39 exerted knockdown effects on USP39 expression.

USP39 knockdown inhibits CRC cell viability and colony formation. To determine the role of USP39 expression on CRC cell viability, an MTT assay was performed on SW1116 and HCT116 cells. As included in Fig. 3A, the viability of cells of SW1116 cells in the KD-1 $(\mathrm{P}<0.0001)$ and KD-2 $(\mathrm{P}<0.001)$ groups was significantly decreased compared with the shCon and Con groups. A similar result was achieved in HCT116 cells (Fig. 3B; both $\mathrm{P}<0.0001$ ). KD-1 suppressed cell viability to a greater extent than KD-2 in the SW1116 and in HCT116 cells, and was therefore selected for use in further procedures.

The colony formation capacity of SW1116 and HCT116 cells was then determined for Con, shCon and KD-1 cells. Representative images of the size of each colony and the number of colonies per well are provided in Fig. 4. Analysis indicated that the number of colonies was reduced in the KD-1 
A
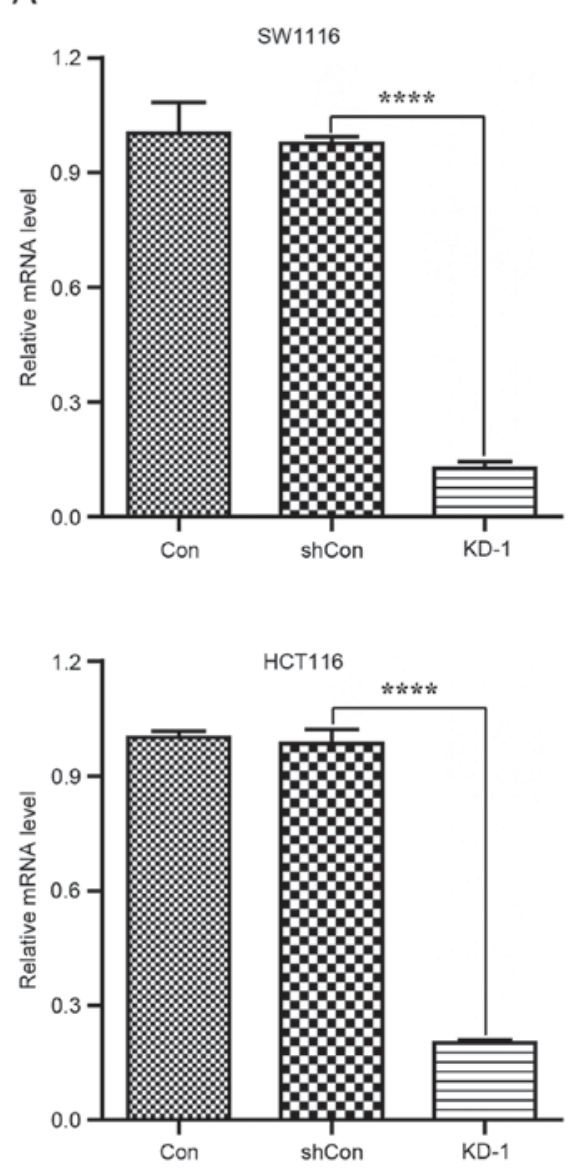

B

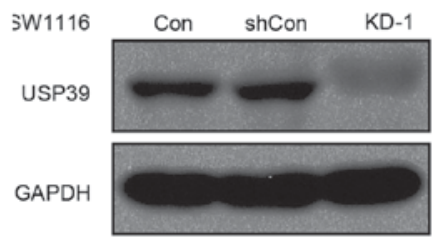

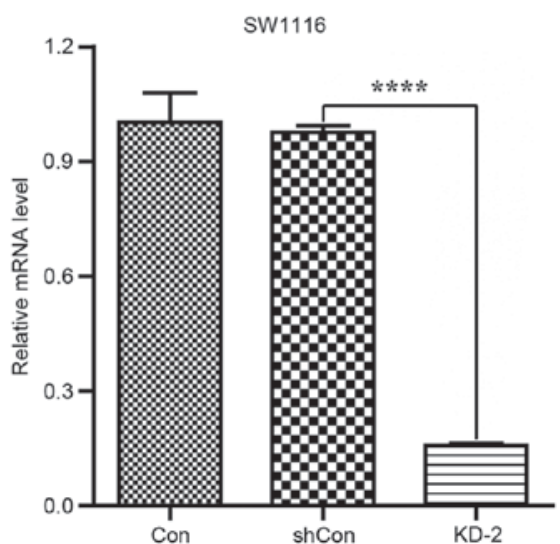
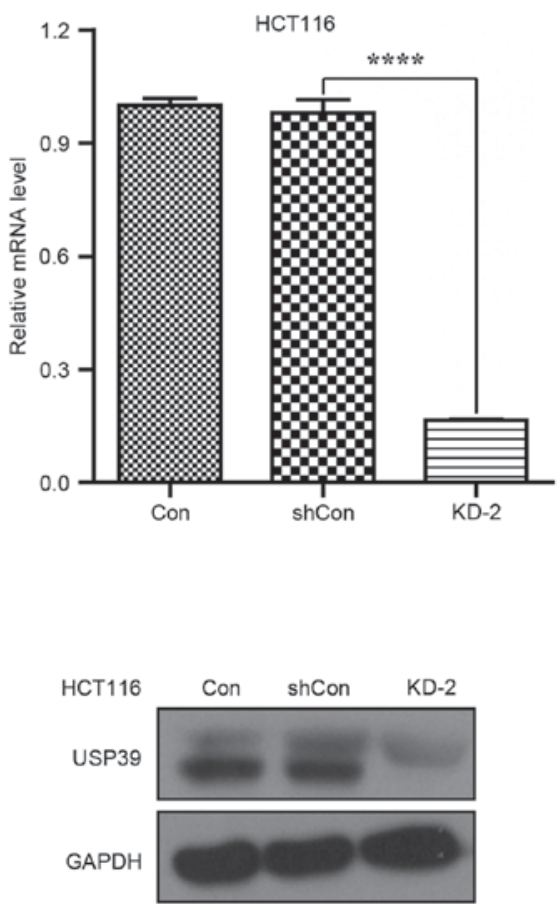

Figure 2. Expression of USP39 was suppressed in SW1116 and HCT116 cells following USP39 knockdown, as confirmed by (A) reverse transcription-quantitative polymerase chain reaction and (B) western blot analyses. $\beta$-actin was used as a control mRNA and GAPDH as a control protein. ${ }^{* * * *} \mathrm{P}<0.0001 \mathrm{compared}$ with shCon. USP39, ubiquitin-specific peptidase 39; shCon, control small hairpin RNA; Con, untransfected control; KD-1, first small hairpin RNA against USP39; KD-2, second small hairpin RNA against USP39.

group, compared with the Con and shCon groups, for SW1116 and HCT116 (Fig. 4; P<0.0001).

USP39 knockdown inhibits cell cycle progression. Cell proliferation is controlled by cell cycle progression. Therefore, cell cycle distribution was analyzed with FACS in SW1116 and HCT116 cells subsequent to USP39 knockdown (Fig. 5A). As presented in Fig. 5B, the proportion of SW1116 and HCT116 cells in the $\mathrm{G}_{0} / \mathrm{G}_{1}(\mathrm{SW} 1116, \mathrm{P}<0.0001 ;$ HCT116, $\mathrm{P}<0.05)$ and $\mathrm{S}$ (both $\mathrm{P}<0.01$ ) phases was significantly reduced, and the proportion in the $\mathrm{G}_{2} / \mathrm{M}$ phase significantly increased (both $\mathrm{P}<0.0001)$ in the KD-1 group compared with the Con and shCon groups. These results indicated that USP39 may have regulated cell proliferation through an effect on cell cycle distribution. In addition, more cells in the sub- $\mathrm{G}_{1}$ phase and therefore, undergoing apoptosis, were detected in SW1116 and
HCT116 cells subsequent to USP39 knockdown (Fig. 5C; both $\mathrm{P}<0.0001)$.

USP39 knockdown promotes apoptosis. To further investigate the apoptosis-promoting effects of USP39 silencing in CRC cells, SW1116 cells were analyzed with Annexin V-APC/7-AAD double staining and flow cytometry (Fig. 6A). As demonstrated in Fig. 6B, the knockdown of USP39 increased the proportion of cells at early (Annexin $\mathrm{V}^{+} / 7-\mathrm{AAD}$-) and late (Annexin $\mathrm{V}^{+} / 7-\mathrm{AAD}^{+}$) apoptotic stages by $\sim 2$-fold compared with the Con and shCon groups $(\mathrm{P}<0.0001)$. This data demonstrated that USP39 silencing induced apoptosis in SW1116 cells. In addition, the expression levels of apoptotic markers, including PARP, p53 and caspase-3, were also assessed in SW1116 cells. As depicted in Fig. 6C, the expression levels of p53, p-p53, PARP and caspase-3 were increased in SW1116 
A
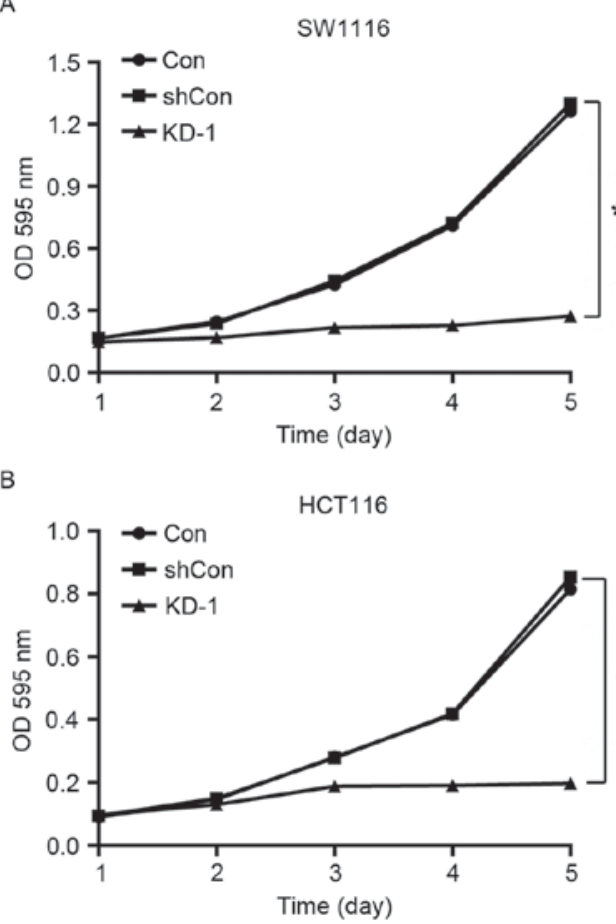
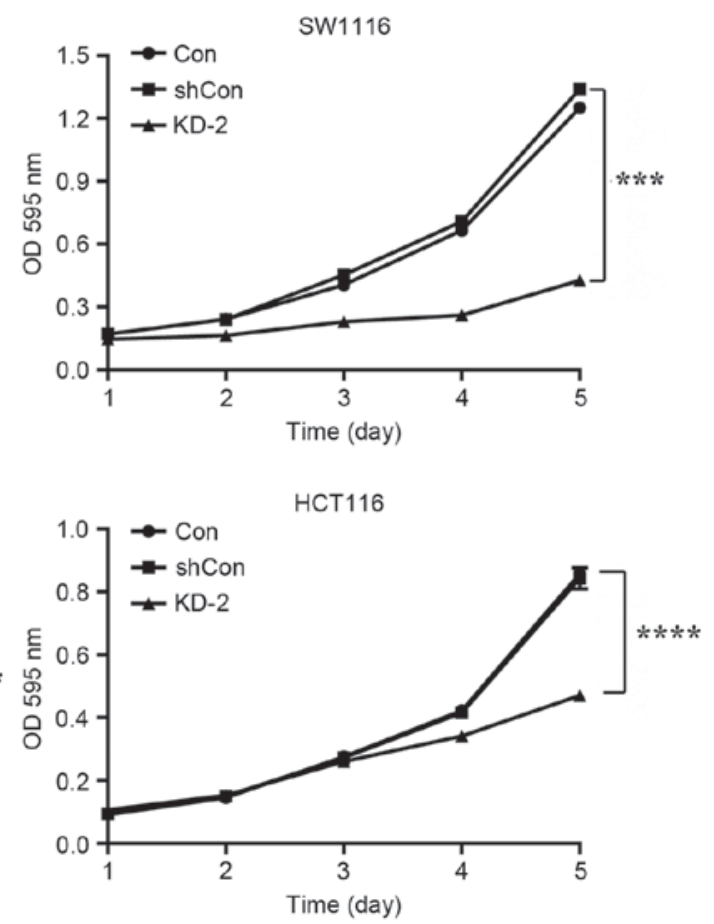

Figure 3. Infection with KD-1 or KD-2 inhibited the viability of SW1116 and HCT116 cells as determined by an MTT assay. The growth of (A) SW1116 and (B) HCT116 cells was significantly reduced following infection with KD-1 or KD-2. Data are presented as the mean \pm standard deviation. ${ }^{* * *} \mathrm{P}<0.001$, ${ }^{* * * * *} \mathrm{P}<0.0001$ compared with shCon. KD-1, first small hairpin RNA against ubiquitin-specific peptidase 39; KD-2, second small hairpin RNA against ubiquitin-specific peptidase 39; shCon, control small hairpin RNA; Con, untransfected control.

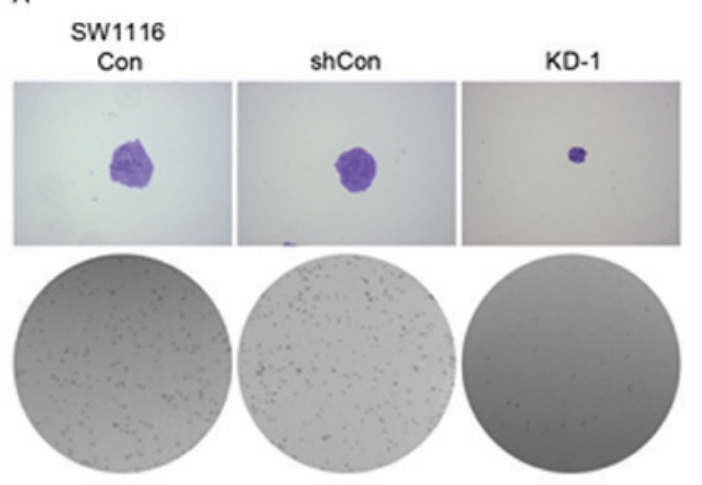

c

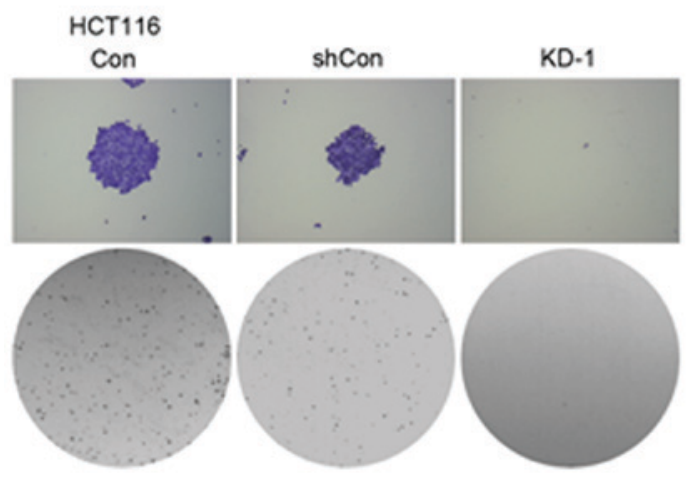

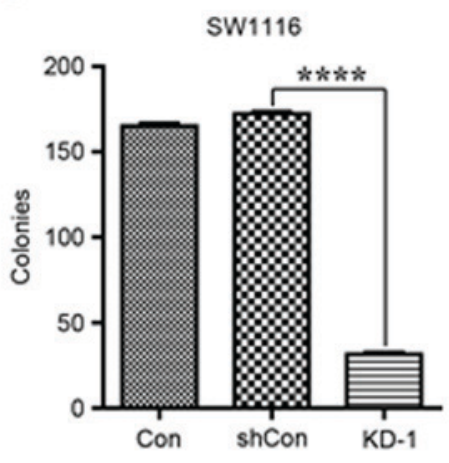

D

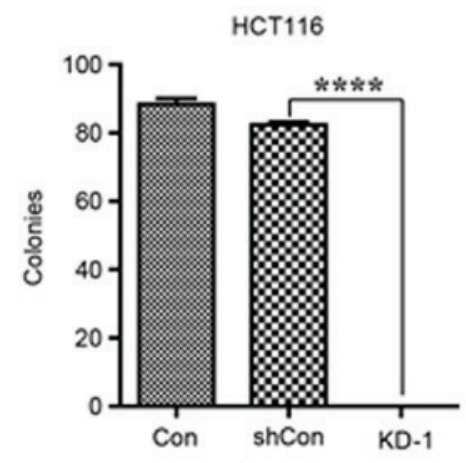

Figure 4. USP39 knockdown impaired the colony formation ability of SW1116 and HCT116 cells. (A) Representative images of the size and number, and (B) quantification of the number of colonies in SW1116 cells. (C) Representative images of the size and number, and (D) quantification of the number of colonies in HCT116 cells. The colony size and number was reduced subsequent to infection with KD-1. Data are presented as the mean \pm standard deviation. ${ }_{* * * * *}^{*}<0.0001$ compared with shCon. USP39, ubiquitin-specific peptidase 39; shCon, control small hairpin RNA; KD-1, first small hairpin RNA against USP39; Con, untransfected control. 
A
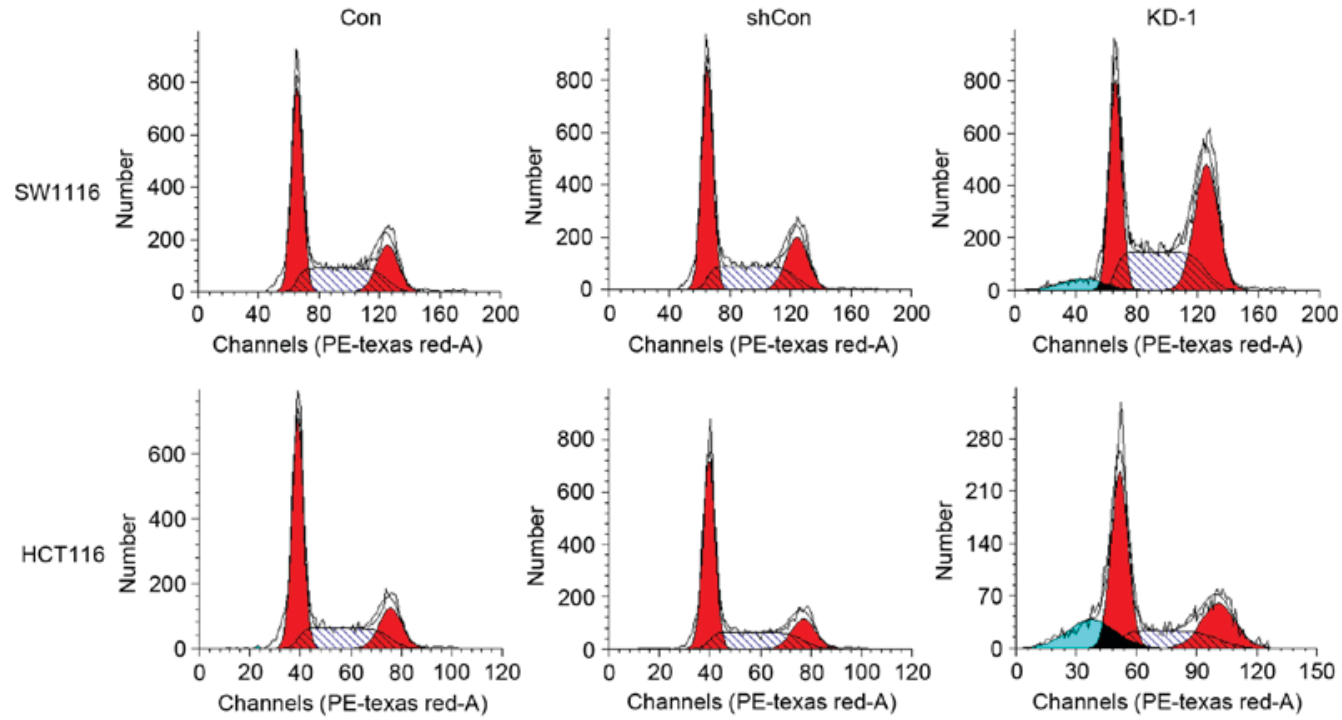

B

SW1116

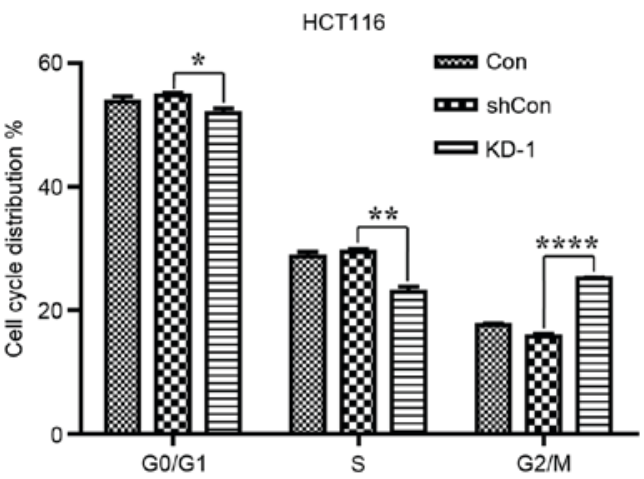

C
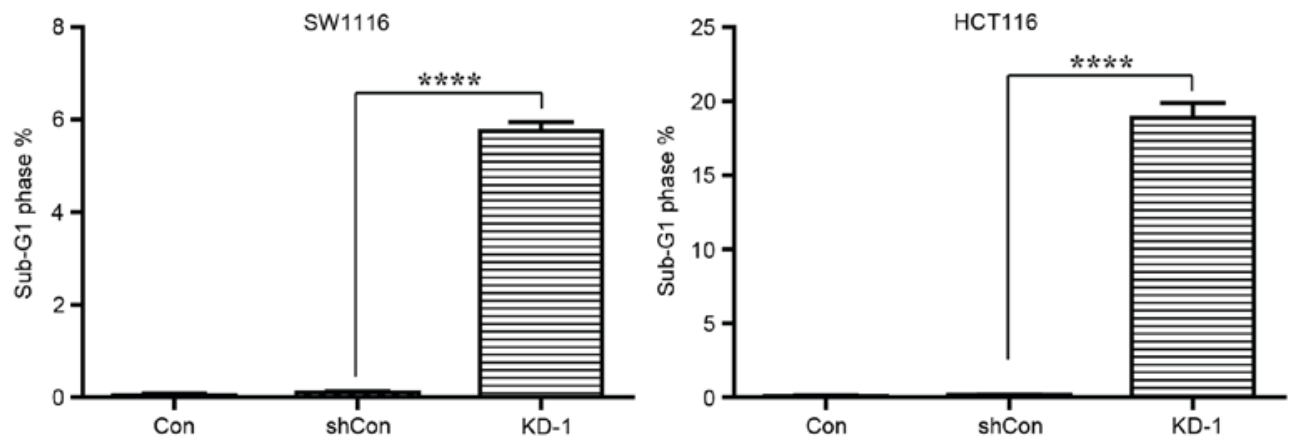

Figure 5. USP39 knockdown inhibited cell cycle progression in SW1116 and HCT116 cells. (A) Flow cytometry analysis of the cell cycle distribution in SW1116 and HCT116 cells. (B) USP39 knockdown led to an increase of cells in the $\mathrm{G}_{2} / \mathrm{M}$ phase, and a decrease in the $\mathrm{G}_{0} / \mathrm{G}_{1}$ and $\mathrm{S}$ phases. (C) USP39 knockdown increased the proportion of cells in the sub- $\mathrm{G}_{1}$ phase. Data are presented as the mean \pm standard deviation. ${ }^{*} \mathrm{P}<0.05,{ }^{* * *} \mathrm{P}<0.01,{ }^{* * * * *} \mathrm{P}<0.0001$ compared with shCon. USP39, ubiquitin-specific peptidase 39; shCon, control small hairpin RNA; KD-1, first small hairpin RNA against USP39; Con, untransfected control.

cells following KD-1 infection. Thus, it was demonstrated that the knockdown of USP39 in SW1116 cells induced apoptosis via altering the expression of apoptosis-associated proteins.

\section{Discussion}

CRC is one of the leading causes of cancer mortality, and results from uncontrolled cell growth in the colon, rectum or appendix (14). Molecular-targeted therapies may present a powerful treatment option for CRC. Thus, the present study focused on the identification of an oncogenic target in $\mathrm{CRC}$ and investigated the biological effects of silencing the identified gene.

In the present study, it was demonstrated that the knockdown of USP39 by USP39-specific shRNA could significantly suppress the growth and colony formation abilities of the CRC cell lines SW1116 and HCT116. It was previously identified that spliceosome factors are associated 
A

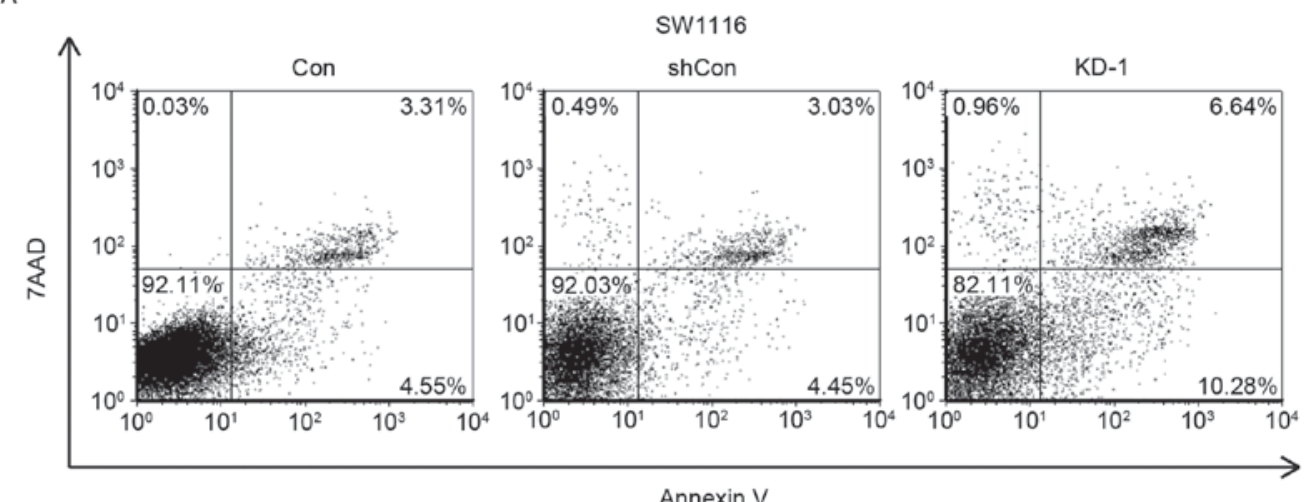

B

SW1116

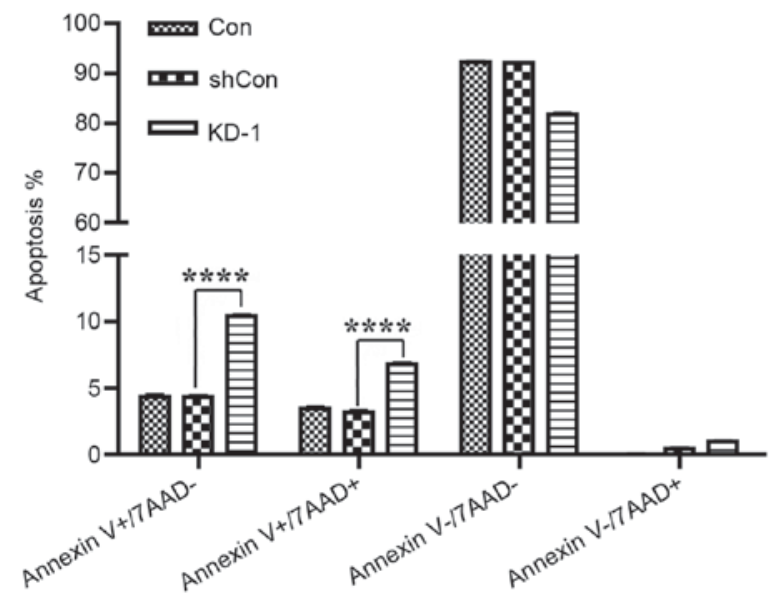

C

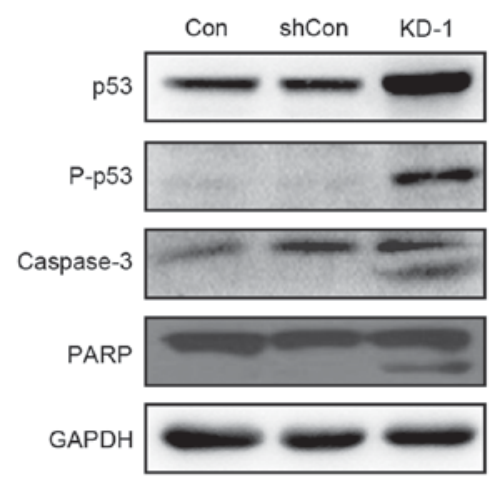

Figure 6. USP39 knockdown promotes apoptosis in SW1116 cells, as assessed by flow cytometry and western blotting analyses. (A) Representative images of Annexin V/7-ADD staining results in SW1116 cells. (B) Quantification of part A. Data are presented as the mean \pm standard deviation. (C) Expression of proteins associated with apoptosis in SW1116 cells as determined by western blot analysis. ${ }^{* * * *} \mathrm{P}<0.0001$ compared with shCon. USP39, ubiquitin-specific peptidase 39; shCon, control small hairpin RNA; KD-1, first small hairpin RNA against USP39; Con, untransfected control; p-, phosphorylated; PARP, poly(ADP-ribose) polymerase.

with cancer development $(15,16)$. As a confirmed spliceosome factor, USP39 is critical to maintain the spindle checkpoint and promote successful cytokinesis through the regulation of Aurora B mRNA splicing in mammalian cells (7). The downregulation of Aurora B leads to defects in spindle checkpoint function and cytokinesis (7). This indicates that USP39 may affect proliferation via affecting Aurora B mRNA splicing. In addition, flow cytometry analysis in the present study identified that the knockdown of USP39 induced cell cycle arrest in the $\mathrm{G}_{2} / \mathrm{M}$ phases, which may have induced the inhibition of proliferation. These results correspond with a previous study, which indicated that the knockdown of USP39 markedly suppressed the proliferation of TT medullary thyroid carcinoma cells (11).

It was identified in the present study that cells accumulated in the sub- $\mathrm{G}_{1}$ phase, indicative of the induction of apoptosis. Further analysis confirmed that USP39 silencing significantly promoted the apoptosis of SW1116 cells. Apoptosis is a caspase-dependent form of programmed cell death (17), which is necessary for the maintenance of bodily health (18). Caspase-3 is the key enzyme in the process of apoptosis (19); once activated, it cleaves specific substrates, including PARP, to mediate apoptosis $(20,21)$. The transcription factor p53, a tumor suppressor, is phosphorylated and activated as a reaction to DNA damage, leading to growth arrest and the induction of cell death (22). Consistent with the results of the present study, the level of p53 expression and PARP cleavage have been demonstrated to increase during the cell cycle arrest and apoptosis of CRC cells (23). Taken together, it can be concluded that the growth inhibition associated with USP39 silencing in CRC cells may have been induced by the induction of spliceosome factor-associated apoptosis.

In conclusion, the present study has revealed that USP39 silencing suppressed CRC cell proliferation via activating the caspase cascade and upregulating the expression of $\mathrm{p} 53$. These data may provide an experimental basis for the development of USP39 as a potential molecular target against CRC. Further investigation is required to verify the efficacy of USP39-targeted therapy in vivo.

\section{Acknowledgements}

Not applicable.

\section{Funding}

No funding was received. 


\section{Availability of data and materials}

The datasets used and/or analyzed during the current study are available from the corresponding author on reasonable request.

\section{Authors' contributions}

ZX performed the experiments and drafted the manuscript. FS and WH participated in the experiments. ZW and XS participated in the data analysis and figure format. FZ participated in the research design, the article reviewing and the data examining.

\section{Ethics approval and consent to participate}

Not applicable.

\section{Consent for publication}

Not applicable.

\section{Competing interests}

The authors declare that they have no competing interests.

\section{References}

1. Gansler T, Ganz PA, Grant M, Greene FL, Johnstone P, Mahoney M, Newman LA, Oh WK, Thomas CR Jr, Thun MJ, et al: Sixty years of CA: A cancer journal for clinicians. CA Cancer J Clin 60: 345-350, 2010.

2. Li Q, Zhou S, Jing J, Yang T, Duan S, Wang Z, Mei Q and Liu L: Oligosaccharide from apple induces apoptosis and cell cycle arrest in HT29 human colon cancer cells. Int J Biol Macromol 57: 245-254, 2013.

3. Allgayer $\mathrm{H}$ and Fulda $\mathrm{S}$ : An introduction to molecular targeted therapy of cancer. Adv Med Sci 53: 130-138, 2008.

4. Lygerou Z, Christophides G and Seraphin B: A novel genetic screen for snRNP assembly factors in yeast identifies a conserved protein, Sad1p, also required for pre-mRNA splicing. Mol Cell Biol 19: 2008-2020, 1999.

5. Hadjivassiliou H, Rosenberg OS and Guthrie C: The crystal structure of S. cerevisiae Sad1, a catalytically inactive deubiquitinase that is broadly required for pre-mRNA splicing. RNA 20: 656-669, 2014.

6. Makarova OV, Makarov EM and Luhrmann R: The 65 and $110 \mathrm{kDa}$ SR-related proteins of the U4/U6.U5 tri-snRNP are essential for the assembly of mature spliceosomes. EMBO J 20: 2553-2563, 2001.

7. van Leuken RJ, Luna-Vargas MP, Sixma TK, Wolthuis RM and Medema RH: Usp39 is essential for mitotic spindle checkpoint integrity and controls mRNA-levels of aurora B. Cell Cycle 7: 2710-2719, 2008.
8. Wen D, Xu Z, Xia L, Liu X, Tu Y, Lei H, Wang W, Wang T, Song L, Ma C, et al: Important role of SUMOylation of Spliceosome factors in prostate cancer cells. J Proteome Res 13: 3571-3582, 2014.

9. Wang H, Ji X, Liu X, Yao R, Chi J, Liu S, Wang Y, Cao W and Zhou Q: Lentivirus-mediated inhibition of USP39 suppresses the growth of breast cancer cells in vitro. Oncol Rep 30: 2871-2877, 2013.

10. Pan Z, Pan H, Zhang J, Yang Y, Liu H, Yang Y, Huang G, Ni J, Huang $J$ and Zhou W: Lentivirus mediated silencing of ubiquitin specific peptidase 39 inhibits cell proliferation of human hepatocellular carcinoma cells in vitro. Biol Res 48: 18, 2015.

11. An Y, Yang S, Guo K, Ma B and Wang Y: Reduced USP39 expression inhibits malignant proliferation of medullary thyroid carcinoma in vitro. World J Surg Oncol 13: 255, 2015.

12. Sun W, Yao L, Jiang B, Guo L and Wang Q: Spindle and kinetochore-associated protein 1 is overexpressed in gastric cancer and modulates cell growth. Mol Cell Biochem 391: 167-174, 2014.

13. Livak KJ and Schmittgen TD: Analysis of relative gene expression data using real-time quantitative PCR and the 2(-Delta Delta C(T)) method. Methods 25: 402-408, 2001.

14. de-Freitas Junior JC and Morgado-Diaz JA: The role of N-glycans in colorectal cancer progression: Potential biomarkers and therapeutic applications. Oncotarget 7: 19395-19413, 2016.

15. Wang L, Lawrence MS, Wan Y, Stojanov P, Sougnez C, Stevenson K, Werner L, Sivachenko A, DeLuca DS, Zhang L, et al: SF3B1 and other novel cancer genes in chronic lymphocytic leukemia. N Engl J Med 365: 2497-2506, 2011.

16. Cazzola M, Rossi M, Malcovati L and Associazione Italiana per la Ricerca sul Cancro Gruppo Italiano Malattie Mieloproliferative: Biologic and clinical significance of somatic mutations of SF3B1 in myeloid and lymphoid neoplasms. Blood 121: 260-269, 2013.

17. Salvesen GS and Dixit VM: Caspases: Intracellular signaling by proteolysis. Cell 91: 443-446, 1997.

18. Shimizu S, Yoshida T, Tsujioka M and Arakawa S: Autophagic cell death and cancer. Int J Mol Sci 15: 3145-3153, 2014.

19. Fan TJ, Han LH, Cong RS and Liang J: Caspase family proteases and apoptosis. Acta Biochim Biophys Sin (Shanghai) 37: 719-727, 2005

20. Ghavami S, Hashemi M, Ande SR, Yeganeh B, Xiao W, Eshraghi M, Bus CJ, Kadkhoda K, Wiechec E, Halayko AJ and Los M: Apoptosis and cancer: Mutations within caspase genes. J Med Genet 46: 497-510, 2009.

21. Matuo R, Sousa FG, Escargueil AE, Grivicich I, Garcia-Santos D, Chies JA, Saffi J, Larsen AK and Henriques JA: 5-Fluorouracil and its active metabolite FdUMP cause DNA damage in human SW620 colon adenocarcinoma cell line. J Appl Toxicol 29: 308-316, 2009.

22. Vousden KH and Lane DP: p53 in health and disease. Nat Rev Mol Cell Biol 8: 275-283, 2007.

23. Thorenoor N, Faltejskova-Vychytilova P, Hombach S, Mlcochova J, Kretz M, Svoboda M and Slaby O: Long non-coding RNA ZFAS1 interacts with CDK1 and is involved in p53-dependent cell cycle control and apoptosis in colorectal cancer. Oncotarget 7: 622-637, 2016.

This work is licensed under a Creative Commons Attribution-NonCommercial-NoDerivatives 4.0 International (CC BY-NC-ND 4.0) License. 